\title{
Potenciais evocados auditivos de tronco encefálico por frequência específica e de estado estável na audiologia pediátrica: estudo de caso
}

\author{
Frequency-specific and steady-state evoked auditory brainstem \\ responses in pediatric audiology: case study
}

\author{
Gabriela Ribeiro Ivo Rodrigues ${ }^{1}$, Mabel Gonçalves Almeida², Dóris Ruth Lewis ${ }^{3}$
}

\begin{abstract}
RESUMO
Preconiza-se o diagnóstico até os três meses de idade em crianças com deficiência auditiva congênita. Após a etapa inicial de confirmação do diagnóstico, é necessário que se obtenha limiares precisos nas diferentes frequências, para que seja possível uma adequada seleção, indicação e regulagem de aparelhos de amplificação sonora. Nesse contexto, inserem-se os Potenciais Evocados Auditivos de Tronco Encefálico por Frequência Específica (PEATE-FE) e, mais recentemente, os Potenciais Evocados Auditivos de Estado Estável (PEAEE). O objetivo deste estudo de caso foi apresentar os achados das duas técnicas para estimar os limiares auditivos em uma criança de três meses de idade, com perda auditiva neurossensorial bilateral, diagnosticada utilizando-se como primeiro método de avaliação os Potenciais Evocados Auditivos de Tronco Encefálico com estímulo clique, tanto por via aérea como por via óssea. As duas técnicas mostraram-se eficientes para estimar os limiares auditivos, com uma vantagem dos PEAEE com relação ao tempo de duração de exame.
\end{abstract}

Descritores: Potenciais evocados auditivos; Potenciais evocados auditivos do tronco encefálico; Eletrofisiologia; Perda auditiva neurossensorial/congênito; Relatos de casos

\section{INTRODUÇÃO}

Com o advento da triagem auditiva neonatal (TAN), crianças cada vez menores são encaminhadas para diagnóstico audiológico, aumentando a necessidade de avaliações mais objetivas e precisas, principalmente no que se refere às estimativas de limiares nas frequências específicas, para posterior seleção e adaptação do aparelho de amplificação sonora individual (AASI).

Recomenda-se que o diagnóstico infantil seja realizado antes dos três de meses de idade e que se utilize uma combi-

Trabalho desenvolvido no Centro Audição na Criança da Divisão de Educação e Reabilitação dos Distúrbios da Comunicação da Pontifícia Universidade Católica de São Paulo - CeAC/DERDIC/PUC-SP - São Paulo (SP), Brasil. (1) Pós-graduanda (Mestrado) do Programa de Pós-graduação em Fonoaudiologia da Pontifícia Universidade Católica de São Paulo - PUC-SP - São Paulo (SP), Brasil.

(2) Pós-graduanda (Mestrado) do Programa de Pós-graduação em Fonoaudiologia da Pontifícia Universidade Católica de São Paulo - PUC-SP - São Paulo (SP), Brasil.

(3) Doutora, Professora Titular da Faculdade de Fonoaudiologia da Pontifícia Universidade Católica de São Paulo - PUC-SP - São Paulo (SP), Brasil. Endereço para correspondência: Gabriela Ribeiro Ivo Rodrigues. CeAC/ DERDIC/PUC-SP. R. Estado de Israel, 860, Vila Clementino, São Paulo (SP), Brasil, CEP: 04022-040. E-mail: gabrielaivo@ hotmail.com Recebido em: 19/9/2008; Aceito em: 18/7/2009 nação de métodos eletrofisiológicos, eletroacústicos e comportamentais, possibilitando assim uma estimativa dos limiares auditivos de forma mais precisa ${ }^{(1)}$.

Nesse contexto, inserem-se os Potenciais Evocados Auditivos de Tronco Encefálico por Frequência Específica (PEATE-FE) e, mais recentemente, os Potenciais Evocados Auditivos de Estado Estável (PEAEE), uma vez que são capazes de predizer os limiares auditivos com especificidade de frequência. Essas técnicas podem orientar o processo de seleção e adaptação do AASI, essencial para uma habilitação/ reabilitação de qualidade ${ }^{(2)}$.

O PEATE-FE é o atual método recomendado para obter essa informação para neonatos, lactentes e crianças que não cooperaram durante a avaliação comportamental ${ }^{(1)}$. É capaz de predizer os níveis mínimos de resposta auditiva em sujeitos com audição normal, ou com diferentes tipos e graus de perdas auditivas. Os níveis mínimos de resposta obtidos pelos PEATE-FE encontram-se entre 10 e $20 \mathrm{~dB}$ acima das respostas comportamentais ${ }^{(3)}$. No entanto, sua utilização limita-se à pesquisa e registro de apenas uma frequência por vez, e em cada orelha separadamente. A interpretação dos achados é subjetiva e os resultados dependem de um avaliador com habilidade e experiência clínica, tornando-se, portanto, um exame de mais difícil realização( ${ }^{(4)}$. 
Por outro lado, os PEAEE vêm despertando o interesse na clínica audiológica pela possibilidade de estimar a audição com a utilização de múltiplos estímulos simultâneos, permitindo a avaliação das quatro frequências $(500,1000,2000$ e $4000 \mathrm{~Hz})$ em ambas as orelhas ao mesmo tempo. Tem-se dessa forma um registro desses potenciais de forma mais rápida, além de mais objetiva, pois nos PEAEE a detecção das respostas é baseada em testes estatísticos e algoritmos, o que reduz os riscos da interpretação subjetiva( ${ }^{(4)}$.

Estudos nacionais e internacionais têm mostrado boas correlações entre as respostas dos PEAEE e os limiares comportamentais em adultos e crianças com perda auditiva ${ }^{(5-6)}$. Estudo que comparou os PEAEE com os PEATE-FE em adultos ouvintes e com perdas auditivas ${ }^{(7)}$ encontrou níveis mínimos de resposta para os PEATE-FE, em média, $3 \mathrm{~dB}$ mais próximos dos limiares comportamentais que os obtidos pelos PEAEE, exceto nas perdas auditivas em rampa, casos em que os PEAEE foram mais precisos. Em neonatos ouvintes ${ }^{(8)}$, comparando as duas técnicas, foram observadas respostas para os PEATE-FE significantemente melhores que para os PEAEE. No entanto, tal diferença foi considerada um artefato de calibração dos estímulos, visto que, quando os resultados dos PEAEE foram calibrados em dB peNPS (pico equivalente nível de pressão sonora), calibração utilizada nos PEATE-FE, não houve diferença entre as respostas obtidas pelos dois exames. Estudo que comparou a maturação entre as respostas dos PEATE-FE e dos PEAEE $^{(9)}$ demonstrou uma diminuição média de $10 \mathrm{~dB}$ nas respostas dos PEAEE do nascimento a seis semanas de vida, efeito maturacional este não observado nos resultados dos PEATE-FE.

Em geral, os estudos em crianças com perda auditiva têm comparado os PEAEE com os PEATE com estímulo clique, demonstrando boas correlações entre as duas técnicas para as altas frequências ${ }^{(10-11)}$. No entanto, foram poucos os estudos que correlacionaram os PEAEE com os PEATE-FE ${ }^{(11-13)}$ e estes priorizaram somente as frequências baixas: 250 e $500 \mathrm{~Hz}^{(12-13)}$, encontrando correlações significantes entre as duas técnicas.

\section{APRESENTAÇÃO DO CASO CLÍNICO}

Neste estudo, será apresentado um caso em que foram utilizados os PEATE- FE e os PEAEE com finalidade de estimar os limiares auditivos de uma criança de três meses de idade, cujo diagnóstico de perda auditiva neurossensorial moderada bilateral foi obtido por meio do PEATE com estímulo clique, tanto por via aérea, como por via óssea.

A pesquisa foi realizada no Centro Audição na Criança
(CeAC), setor da Divisão de Educação e Reabilitação dos Distúrbios da Comunicação (DERDIC), ambos da Pontifícia Universidade Católica de São Paulo (PUC-SP), com aprovação da instituição e do Comitê de Ética (processo no 113/2008). Os responsáveis pela criança envolvida assinaram o Termo de Consentimento Livre e Esclarecido.

A criança do sexo feminino e com três meses de idade, analisada neste caso, foi encaminhada à instituição para reavaliação audiológica. Apresentava histórico de falha na TAN na maternidade, utilizando o protocolo com Emissões Otoacústicas Evocadas por Estímulo Transiente (EOAET) e PEATE com estímulo clique a $45 \mathrm{~dB}$ NA. O primeiro diagnóstico realizado com um mês de idade, em outra instituição, utilizou os PEATE com estímulo clique por via aérea e óssea e os PEATE-FE, sendo ambos sugestivos de perda auditiva neurossensorial moderada bilateral. Os resultados da avaliação eletrofisiológica inicial foram apresentados no Quadro 1.

$\mathrm{Na}$ reavaliação, foram investigados todos os indicadores de risco para deficiência auditiva ${ }^{(1)}$, sendo constatada história familiar de perda auditiva congênita durante exame médico. Foi solicitada a reavaliação para confirmação dos limiares auditivos, pois os pais suspeitavam de mudança nos limiares da criança. Após avaliação médica e entrevista fonoaudiológica, foi realizada uma bateria de exames audiológicos, descritos a seguir.

Foi realizada a timpanometria com sonda de tom - teste de $1000 \mathrm{~Hz}$, com resultado sugestivo de curva timpanométrica do tipo pico simples ${ }^{(14)}$. O registro das Emissões Otoacústicas Evocadas por Estímulo Transiente (EOAET) e Emissões Otoacústicas Evocadas por Produto de Distorção (EOAE-PD) estavam ausentes bilateralmente.

$\mathrm{Na}$ avaliação comportamental, devido à idade da criança e sua capacidade motora e cognitiva, foi possível realizar a audiometria de observação comportamental (BOA) em campo livre, nos tons puros de 500, 1000, 2000 e $4000 \mathrm{~Hz}$ calibrados segundo a norma ISO 389-1.

Para avaliação eletrofisiológica foi utilizado o equipamento modelo SmartEp da marca Intelligent Hearing Systems ${ }^{\circledR}$ (IHS). Todos os exames eletrofisiológicos foram realizados com a criança em sono natural com o mesmo padrão de colocação dos eletrodos de superfície para os dois testes, a saber: eletrodos referência dispostos nas mastóides direita (A2) e esquerda (A1); os eletrodos ativo (Fz) e terra (Fpz) na fronte.

No registro dos PEATE-FE os estímulos utilizados foram tonepipes de 500,1000, 2000 e $4000 \mathrm{kHz}$ na taxa de repetição de $39.1 \mathrm{~Hz}$ e polaridade condensada. Os níveis mínimos de resposta foram pesquisados a passos de $10 \mathrm{~dB}$ e a presença

Quadro 1. Avaliação com um mês (em dB NA)

\begin{tabular}{|c|c|c|c|c|c|c|}
\hline \multirow[t]{2}{*}{ Teste } & \multicolumn{3}{|c|}{ Orelha direita } & \multicolumn{3}{|c|}{ Orelha esquerda } \\
\hline & $500 \mathrm{~Hz}$ & $1500 \mathrm{~Hz}$ & $4000 \mathrm{~Hz}$ & $500 \mathrm{~Hz}$ & $1500 \mathrm{~Hz}$ & $4000 \mathrm{~Hz}$ \\
\hline PEATE-FE & 70 & 70 & 60 & 50 & 50 & 60 \\
\hline PEATE-clique VA & & & 60 & & & 60 \\
\hline PEATE-clique VO & & & $\downarrow 60$ & & & $\downarrow 60$ \\
\hline
\end{tabular}

Legenda: PEATE-FE = potenciais evocados auditivos de tronco encefálico por frequência específica; PEATE-clique VA = potenciais evocados auditivos de tronco encefálico com o estímulo clique, registrados por via aérea; PEATE VO = potenciais evocados auditivos de tronco encefálico com o estímulo clique, registrados por via óssea 
de resposta foi determinada pelo avaliador, com base na reprodutibilidade da onda $\mathrm{V}$. O número mínimo de estímulos considerado foi 2000, e o máximo 6000 estímulos.

Os níveis mínimos de resposta para os PEAEE foram pesquisados a passos de $5 \mathrm{~dB}$ e detectados automaticamente pelo sistema. Cada estímulo utilizado consistiu da combinação múltipla de quatro tonepipes nas frequências portadoras de 500, 1000, 2000 e $4000 \mathrm{kHz}$, apresentados nas taxas de repetição de aproximadamente 77, 85, 93 e $101 \mathrm{~Hz}$ na orelha esquerda e de 79, 87, 95 e $103 \mathrm{~Hz}$ na orelha direita. O número máximo de estímulos apresentados foi 400. Os PEAEE foram registrados em dB nível de pressão sonora (NPS) e convertidos para dB nível de audição (NA) segundo a norma ISO 389-2 para fones de inserção, com o intuito de viabilizar as comparações. Essa conversão foi realizada em estudo que utilizou o mesmo equipamento ${ }^{(15)}$.

Foi determinado nível mínimo de resposta auditiva, a menor intensidade em que as respostas foram registradas nas duas técnicas. Os resultados encontrados nos potenciais evocados e o tempo gasto para seu registro, assim como os resultados da audiometria de observação comportamental foram apresentados no Quadro 2.

Os traçados obtidos no registro dos PEATE-FE são apresentados na Figura 1 e os níveis mínimos de resposta observados no registro dos PEAEE na Figura 2.

\section{DISCUSSÃO}

Tanto os PEATE-FE como os PEAEE puderam estimar os limiares auditivos, confirmando o diagnóstico de perda auditiva moderada bilateral. Os níveis mínimos de resposta obtidos pelos PEAEE apresentaram-se semelhantes aos do PEATE-FE em ambas as orelhas, embora levemente menores. Esses achados confrontam aqueles observados em estudo que comparou as duas técnicas em adultos ${ }^{(7)}$, que obtiveram respostas melhores para os PEATE-FE, exceto nas perdas auditivas em rampa, em que os PEAEE forneceram uma estimativa mais precisa.

Quadro 2. Avaliação com três meses (em dB NA)

\begin{tabular}{|c|c|c|c|c|c|c|c|c|c|}
\hline \multirow[t]{2}{*}{ Teste } & \multicolumn{4}{|c|}{ Orelha direita } & \multicolumn{4}{|c|}{ Orelha esquerda } & \multirow{2}{*}{$\begin{array}{l}\text { Duração do } \\
\text { exame (min) }\end{array}$} \\
\hline & $500 \mathrm{~Hz}$ & $1000 \mathrm{~Hz}$ & $2000 \mathrm{~Hz}$ & $4000 \mathrm{~Hz}$ & $500 \mathrm{~Hz}$ & $1000 \mathrm{~Hz}$ & $2000 \mathrm{~Hz}$ & $4000 \mathrm{~Hz}$ & \\
\hline PEATE-FE & 60 & 70 & 70 & 80 & 50 & 50 & 60 & 70 & 80 \\
\hline PEATE-clique VA & & 70 & & & & & 50 & & \\
\hline PEAEE & 49 & 55 & 52 & 64 & 54 & 60 & 59 & 59 & 59 \\
\hline BOA & 70 & 75 & 80 & 80 & & & & & \\
\hline
\end{tabular}

Legenda: PEATE-FE = potenciais evocados auditivos de tronco encefálico por frequência específica; PEATE-clique VA = potenciais evocados auditivos de tronco encefálico com estímulo clique, registrados por via aérea; PEAEE = potenciais evocados auditivos de estado estável; BOA = audiometria de observação comportamental; $\min =$ minutos

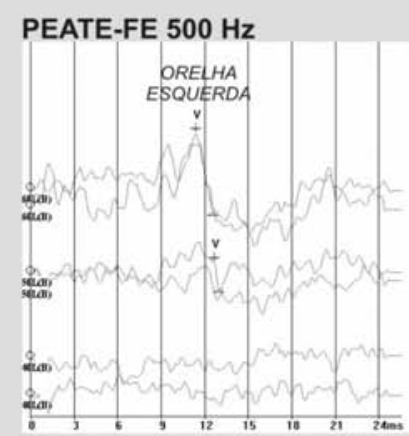

PEATE-FE $2000 \mathrm{~Hz}$

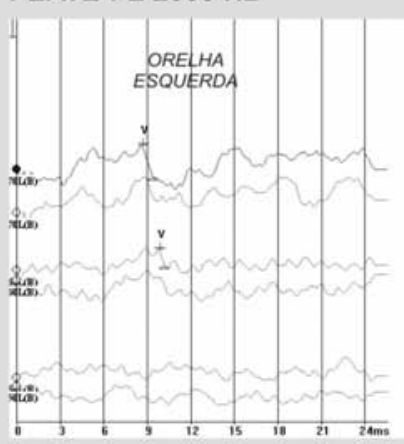

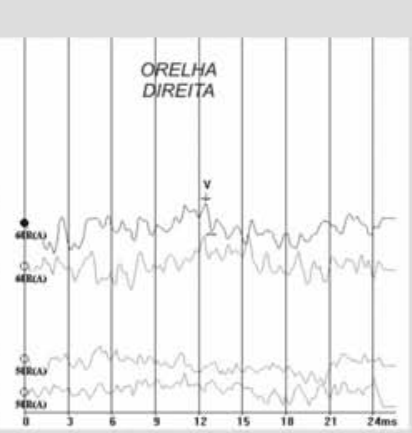

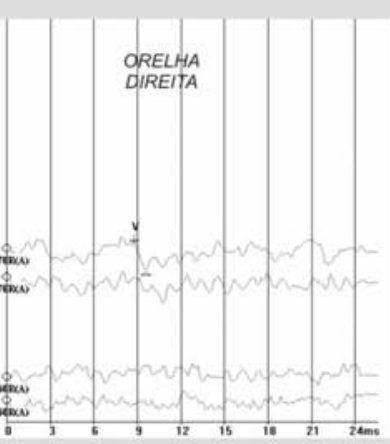

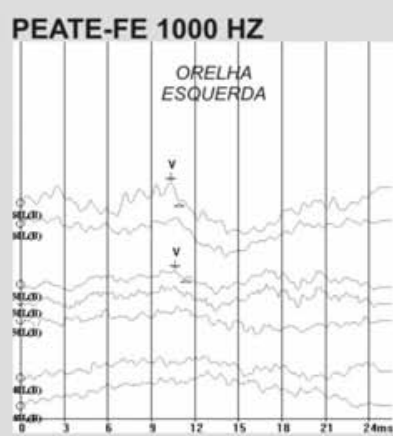
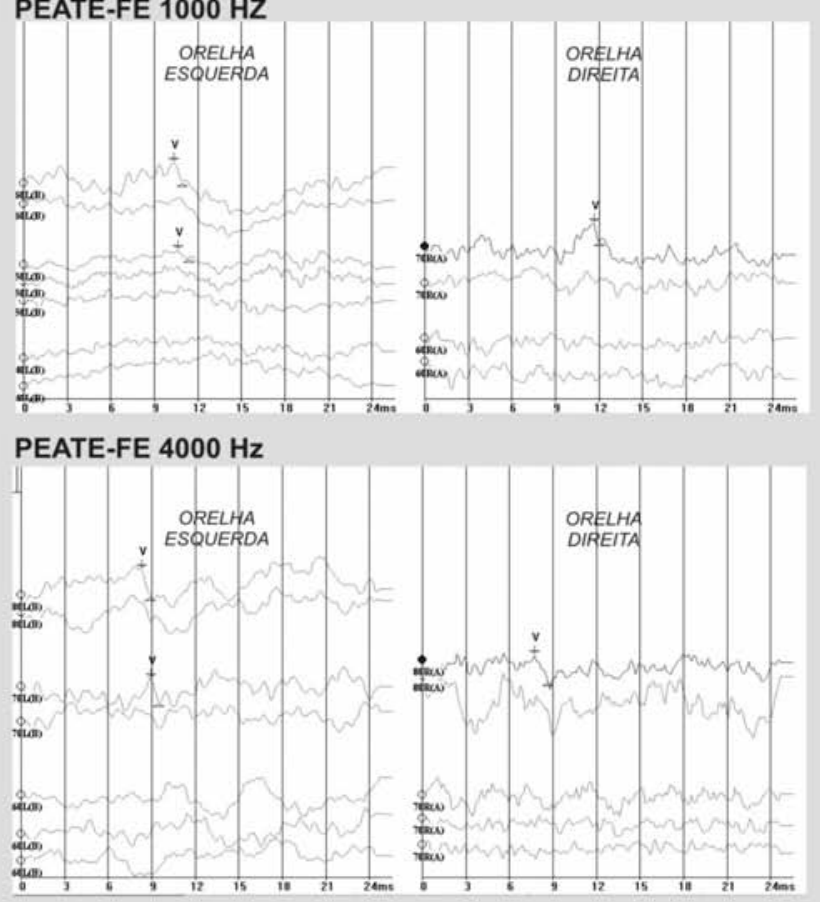

Figura 1. Traçados obtidos no PEATE-FE nas frequências de 500, 1000, 2000 e $4000 \mathrm{~Hz}$ nas orelhas direita e esquerda em dB NA 


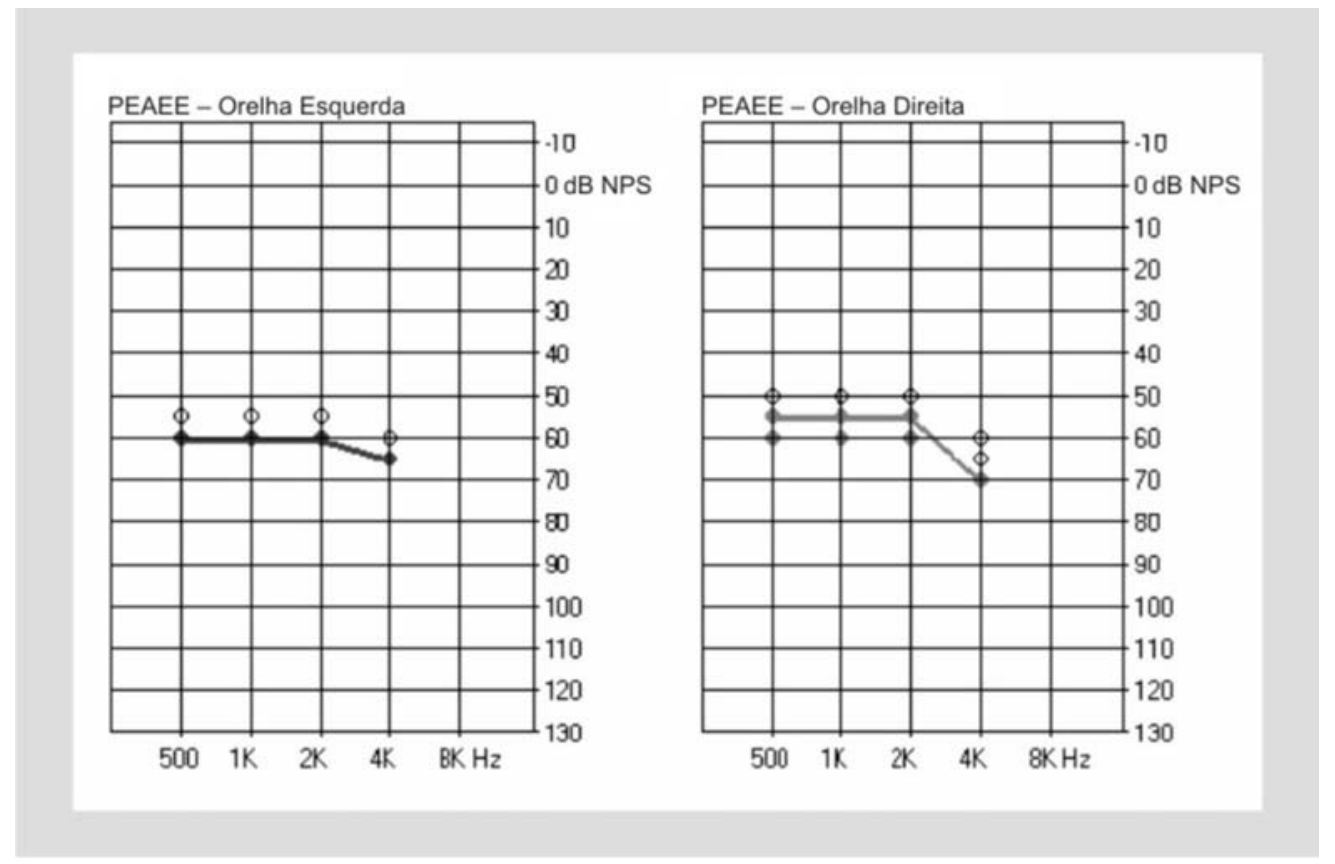

Figura 2. Estimativa do audiograma das orelhas direita e esquerda obtida pelos PEAEE em dB NPS

Comparando a avaliação eletrofisiológica realizada com um mês de idade com a posteriormente realizada aos três meses, notam-se diferenças de aproximadamente $10 \mathrm{~dB}$ tanto nos achados do PEATE - clique como dos PEATE-FE. Tais diferenças podem ser explicadas, possivelmente, pelos diferentes parâmetros de estimulação utilizados nas duas avaliações, pela subjetividade da interpretação das respostas entre dois diferentes avaliadores ${ }^{(4)}$, ou ainda, pela maturação do sistema nervoso auditivo central (SNAC) ${ }^{(8-9)}$.

As diferenças entre os níveis mínimos de resposta obtidos pelas duas técnicas também podem ser justificadas pelas distintas unidades de calibração e formas de registro: PEAEE registrados automaticamente pelo sistema em dB NPS, convertidos para $\mathrm{dB}$ NA e pesquisados a passos de $5 \mathrm{~dB}$; e os PEATE-FE registrados com base na observação do avaliador em dB NA e obtidos a passos de $10 \mathrm{~dB}^{(8-11)}$.

No que concerne ao tempo de realização dos exames, a realização dos PEATE-FE nas frequências de 500, 1000, 2000 e $4000 \mathrm{~Hz}$ em ambas as orelhas durou aproximadamente 80 minutos, enquanto a dos PEAEE durou aproximadamente 59 minutos. Os PEATE-FE demoraram 21 minutos a mais para serem finalizados quando comparados aos PEAEE, o que pode ser justificado pela possibilidade de se utilizar estímulos múltiplos e avaliar as duas orelhas simultaneamente, indicando uma vantagem na utilização dos PEAEE quando comparados aos PEATE-FE ${ }^{(3-4)}$.

\section{CONCLUSÃO}

Este estudo de caso demonstra que as duas medidas eletrofisiológicas apresentam utilidade clínica no diagnóstico da perda auditiva em crianças pequenas que não respondem com precisão a uma avaliação convencional como a audiometria tonal, predizendo os limiares auditivos e, dessa forma, possibilitando o início da seleção e adaptação do AASI antes dos seis meses de idade de forma mais precisa.

\begin{abstract}
It is recommended that congenital hearing loss is identified as early as three months old. After the initial step of confirming the diagnosis, it is necessary to obtain accurate hearing thresholds, allowing an adequate selection, indication and regulation of hearing aids for these children. It is inserted, in this context, the Frequency-Specific Auditory Brainstem Responses (FSABR) and, more recently, the Auditory Steady-State Responses (ASSR). The aim of the present study was to describe the findings of the use of both techniques to estimate the auditory thresholds of a three-month-old infant with bilateral sensorineural hearing loss diagnosed using, as primary evaluation method, the click-evoked Auditory Brainstem Responses, with both air and bone stimuli conduction. Both techniques provided reliable findings for estimating auditory thresholds. The ASSR had an advantage regarding the duration of the evaluation.
\end{abstract}

Keywords: Evoked potentials, auditory; Evoked potentials, auditory, brain stem; Electrophysiology; Hearing loss, sensorineural/ congenital; Case reports 


\section{REFERÊNCIAS}

1. American Academy of Pediatrics, Joint Committee on Infant Hearing. Year 2007 position statement: Principles and guidelines for early hearing detection and intervention programs. Pediatrics. 2007;120(4):898-921.

2. Sininger YS. Audiologic assessment in infants. Curr Opin Otolaryngol Head Neck Surg. 2003;11(5):378-82.

3. Stapells DR. Thresholds estimation by tone-evoked auditory brainstem response: a literature meta-analysis. J Speech Lang Path Audiol. 2000;24(2):74-83.

4. Stapells DR. Frequency-specific evoked potential audiometry in infants. In. Seewald RC: A sound foundation through early amplification. Chicago: Phonak.; 2000. p.13-31.

5. Duarte JL, Alvarenga KF, Garcia TM, Costa Filho OA, Lins OG. A resposta auditiva de estado estável na avaliação auditiva: aplicação clínica. Pró-Fono. 2008;20(2):105-10.

6. Perez-Abalo MC, Savio G, Torres A, Martin V, Rodríguez E, Galán L. Steady state responses to multiple amplitude-modulated tones: an optimized method to test frequency-specific thresholds in hearing-impaired children and normal-hearing subjects. Ear Hear. 2001;22(3):200-11.

7. Johnson TA, Brown CJ. Threshold prediction using the auditory steadystate response and the tone burst auditory brain stem response: a withinsubject comparison. Ear Hear. 2005;26(6):559-76.
8. Rance G, Tomlin D, Rickards FW. Comparison of auditory steady-state responses and tone-burst auditory brainstem responses in normal babies. Ear Hear. 2006;27(6):751-62.

9. Rance G, Tomlin D. Maturation of auditory steady-state responses in normal babies. Ear Hear. 2006;27(1):20-9.

10. Luts H, Desloovere C, Wouters J. Clinical application of dichotic multiple-stimulus auditory steady-state responses in high-risk newborns and young children. Audiol Neurootol. 2006;11(1):24-37.

11. Cone-Wesson B, Dowell RC, Tomlin D, Rance G, Ming WJ. The auditory steady-state response: comparisons with the auditory brainstem response. J Am Acad Audiol. 2002;13(4):173-87.

12. Vander Werff KR, Brown CJ, Gienapp BA, Schimidt Clay KM. Comparison of auditory steady-state response and auditory brainstem response thresholds in children. J Am Acad Audiol. 2002;13(5): 227-35.

13. Stueve MP, O’Rourke C. Estimation of hearing loss in children: comparison of auditory steady-state response, auditory brainstem response, and behavioral test methods. Am J Audiol. 2003;12(2):125-36.

14. Lidén G. The scope and application of current audiometric tests. J Laryngol Otol. 1969;83(6):507-20.

15. Han D, Mo L, Liu H, Chen J, Huang L. Threshold estimation in children using auditory steady-state responses to multiple simultaneous stimuli. ORL J Otorhinolaryngol Relat Spec. 2006;68(2):64-8. 